\title{
EXPLOSION MECHANISMS OF MASSIVE STARS
}

\section{A Critical Review of Possibilities and Perspectives}

\author{
H.-Thomas Janka, \\ Robert Buras, Konstantinos Kifonidis, and Markus Rampp \\ Max-Planck-Institut für Astrophysik, \\ Karl-Schwarzschild-Str. 1, D-85741 Garching, Germany \\ thj@mpa-garching.mpg.de \\ Tomek Plewa \\ Dept. of Astron. and Astrophysics and Center for Astrophysical Thermonuclear Flashes, \\ The University of Chicago, Chicago, IL 60637, U.S.A. \\ and Nicolaus Copernicus Astronomical Center, Bartycka 18, 00716 Warsaw, Poland
}

\begin{abstract}
One of the central problems in supernova theory is the question how massive stars explode. Understanding the physical processes that drive the explosion is crucial for linking the stellar progenitors to the final remnants and for predicting observable properties like explosion energies, neutron star and black hole masses, nucleosynthetic yields, explosion anisotropies, and pulsar kicks. In this article we review different suggestions for the explosion mechanism and discuss the constraints that can or cannot be deduced from observations. The prompt hydrodynamical bounce-shock mechanism has turned out not to work for typical stellar iron cores and empirical values of the compressibility of bulk nuclear matter. Magnetohydrodynamical models on the other hand contain a number of imponderabilities and are still far behind the level of refinement that has been achieved in nonmagnetic simulations. In view of these facts the neutrino-driven mechanism must still be considered as the standard paradigm to explain the explosion of ordinary supernovae, although its viability has yet to be demonstrated convincingly. Since spherically symmetric models do not yield explosions, the hope rests on the helpful effects of convection inside the nascent neutron star, which could boost the neutrino luminosity, and convective overturn in the neutrino-heated region behind the stalled shock, which increases the efficiency of neutrino-energy transfer in this layer. Here we present the first two-dimensional simulations of these processes which have been
\end{abstract}


performed with a Boltzmann solver for the neutrino transport and a state-of-the-art description of neutrino-matter interactions. Although our most complete models fail to explode, convection brings them encouragingly close to a success. An explosion could be obtained by just a minor modification of the neutrino transport, in which case the exploding model fulfills important requirements from observations. We discuss necessary improvements on the route to finally successful models.

Keywords: Supernovae, Neutrinos, Radiation-Hydrodynamics

\section{Introduction}

Supernova explosions of massive stars are powered by the gravitational binding energy that is released when the initial stellar core collapses to a compact remnant and its radius shrinks from typically a few thousand kilometers to little more than ten kilometers. For solar-metallicity progenitors with main-sequence masses of less than about $20-25 \mathrm{M}_{\odot}$ the compact leftover will be neutron star. In case of more massive stars a black hole will be formed, most likely by the fallback - on a timescale of seconds to hours - of matter that does not become unbound in the stellar explosion. But also the direct collapse of the stellar core to a black hole on a multiple of the dynamical timescale is possible (Woosley, Heger, \& Weaver 2002; Heger et al. 2002).

Since the collapse proceeds essentially adiabatically the total energy of the stellar core is conserved during the implosion. The gravitational energy is temporarily stored as internal energy, mainly of degenerate electrons and electron neutrinos. If rotation plays a significant role in the progenitor core, a major fraction of the potential energy may also be converted to rotational energy of the nascent neutron star (or black hole).

The disruption of the massive star in a supernova explosion now means that some fraction of the energy in these reservoirs has to be transferred from the compact central object to the outer stellar layers. What are the physical mechanisms to mediate this energy transfer to the ejecta? And on what timescale do they work? Proposals in the literature include the hydrodynamical bounce-shock, neutrinos, or magnetic fields. The former would initiate the explosion on a dynamical timescale, whereas the latter two can establish the energy transfer only on the secular timescales of neutrino diffusion or magnetic field amplification, respectively.

Unfortunately, observations have so far not been able to yield direct insight into the processes in the stellar center at the onset of the explosion. The hope is that a future Galactic supernova will change this situation by allowing the measurements of a large number of neutrinos and possibly of a gravitational wave signal in great detail. The few neu- 
trino events discovered in connection with Supernova 1987A were a clear signal of stellar core collapse and neutron star formation, but they were not sufficient to reveal the secrets of the explosion. Up to now we have to exploit the less direct information that is provided by the supernova light, by the characteristic properties of supernovae and diffuse and compact supernova remnants, and by the nucleosynthesis of heavy elements which takes place in the immediate vicinity of the newly formed neutron star.

Section 2 will discuss the constraints for the explosion mechanism that are associated with such observations. In Sect. 3 we shall briefly review the different suggestions that have been brought forward to explain the explosions of massive stars and will critically evaluate our knowledge on grounds of theoretical considerations. In Sect. 4 we shall summarize the status of detailed hydrodynamical supernova simulations and their implications for our understanding of the delayed explosion mechanism by neutrino-energy deposition behind the supernova shock. In Sect. 5 we shall present the first results of a new generation of multi-dimensional supernova models which employ a Boltzmann solver for the neutrino transport and a state-of-the-art description of neutrino-matter interac-

tions. Section 6 will conclude with an appraisal of the results and an outlook on open ends.

\section{Observational Facts}

Progress in our understanding of the processes that lead to the explosion of massive stars is mainly based on elaborate numerical modeling, supplemented by theoretical analysis and constrained by a growing data base of observed properties of supernovae. The latter may carry imprints from the physical conditions very close to the center of the explosion. Observable features at very large radii, however, can be linked to the actual energy source of the explosion only indirectly through a variety of intermediate steps and processes. Any interpretation with respect to the mechansim that initiates the explosion therefore requires caution.

A viable model for the explosion mechanism of massive stars should ultimately be able to explain the observed explosion energies, nucleosynthetic yields (in particular of radioactive isotopes like ${ }^{56} \mathrm{Ni}$, which are created near the mass cut), and the masses of the compact remnants (neutron stars or black holes) and their connection with the progenitor mass.

Recent evaluations of photometric and spectroscopic data for samples of well-observed Type-II plateau supernovae reveal a wide continuum of kinetic energies and ejected nickel masses. Faint, low-energy cases 

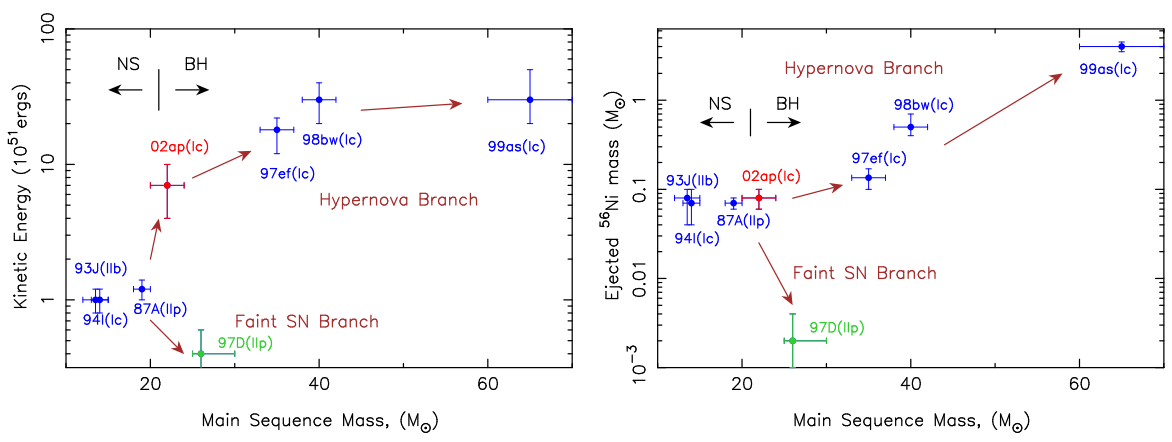

Figure 1. Explosion energy (left) and ejected ${ }^{56} \mathrm{Ni}$ mass as functions of the main sequence mass of the progenitor stars for several supernovae and hypernovae [from Nomoto et al. (2002)].

seem to be nickel-poor whereas bright, high-energy explosions tend to be nickel-rich and associated with more massive progenitors (Hamuy 2002). This direct correlation between stellar and explosion properties, however, is not apparent in an independent analysis by Nadyozhin (2002) who speculates that more than one stellar parameter (rotation or magnetic fields besides the progenitor and core mass) might determine the explosion physics. A large range of nickel masses and explosion energies was also found for Type Ib/c supernovae (Hamuy 2002). Interpreting results obtained by the fitting of optical lightcurves and spectra, Nomoto et al. (2002) proposed that explosions of stars with main sequence masses above $20-25 \mathrm{M}_{\odot}$ split up to a branch of extraordinarily bright and energetic events ("hypernovae") at the one extreme and a branch of faint, low-energy or even "failed" supernovae at the other. Stars with such large masses might collapse to black holes rather than neutron stars. The power of the explosion could depend on the amount of angular momentum in the collapsing core, which in turn can be sensitive to a number of effects such as stellar winds and mass loss, metallicity, magnetic fields, binarity or spiraling-in of a companion star in a binary system.

Another constraint for supernova theory comes from measured neutron star and black hole masses. A direct mass determination may not be possible for compact remnants inside supernova explosions, but the combined information about progenitor (ejecta) mass, kinetic energy, and nickel mass of a supernova (as deduced from spectroscopic and photometric data) may be used to determine the mass cut in a progenitor model by reproducing the known parameters with numerical simulations. Masses of binary pulsars (Thorsett \& Chakrabarty 1999; Stairs et al. 2002) and X-ray binaries (Bailyn et al. 1998) also add to our knowledge about compact supernova remnants. In this case, however, binary 
effects make it even more difficult to establish a link to progenitor properties and therefore conclusions on the supernova mechanism should be drawn with great caution.

Complete and incomplete silicon and oxygen burning behind the supernova shock depend on the strength of the shock and the structure of the progenitor. The abundances of corresponding nucleosynthetic products, if observed in an individual supernova or supernova remnant, can therefore yield information about the exploding star and the explosion energy. Their spatial distribution can also reveal anisotropies and mixing phenomena during the explosion as in case of, e.g., Supernova 1987A or the Vela supernova remnant. Another serious constraint for the explosion mechanism and the conditions near the mass cut comes from a comparison of integral yields of supernova nucleosynthesis with Galactic abundances. The ejected mass of closed-neutron shell isotopes with $N=50$ of $\mathrm{Sr}, \mathrm{Y}$, and $\mathrm{Zr}$, for example, is limited to less than about $10^{-4} \mathrm{M}_{\odot}$ per event [Hoffman et al. (1996)]. These stable nuclei are easily created through the capturing of $\alpha$-particles in the neutron-rich matter around the mass cut and thus experience conditions in the close vicinity of the neutron star. The crucial parameters are entropy and neutron excess. Both are determined by the exposure to high neutrino fluxes, because neutrinos exchange energy and lepton number with the stellar medium.

Anisotropic processes and large-scale mixing between the deep interior and the hydrogen layer had to be invoked in case of Supernova 1987A to explain the shape of the lightcurve, the unexpectedly early appearance of X-ray and $\gamma$-ray emission, and Doppler features of spectral lines (for a review, see Nomoto et al. 1994). Fifteen years after the explosion, the expanding debris exhibits an axially symmetric deformation (Wang et al. 2002). Supernova 1987A therefore seems to possess an intrinsic, global asymmetry. The same conclusion was drawn for other core-collapse supernovae (Type-II as well as Ib/c) based on the fact that their light is linearly polarized at a level around $1 \%$ with a tendency to increase at later phases when greater depths are observed (Wang et al. 2001; Leonhard et al. 2001). This has been interpreted as evidence that the inner portions of the explosion, and hence the mechanism itself, are strongly non-spherical (Höflich, Wheeler, \& Wang 1999; Wheeler et al. 2000), possibly associated with a "jet-induced" explosion (Wang et al. 2002; Khokhlov et al. 1999). This is a very interesting and potentially relevant conjecture. It does, however, not necessarily constrain the nature of the physical process that mediates the energy transfer from the collapsed core of the star to the ejecta and thereby creates the asphericity. 

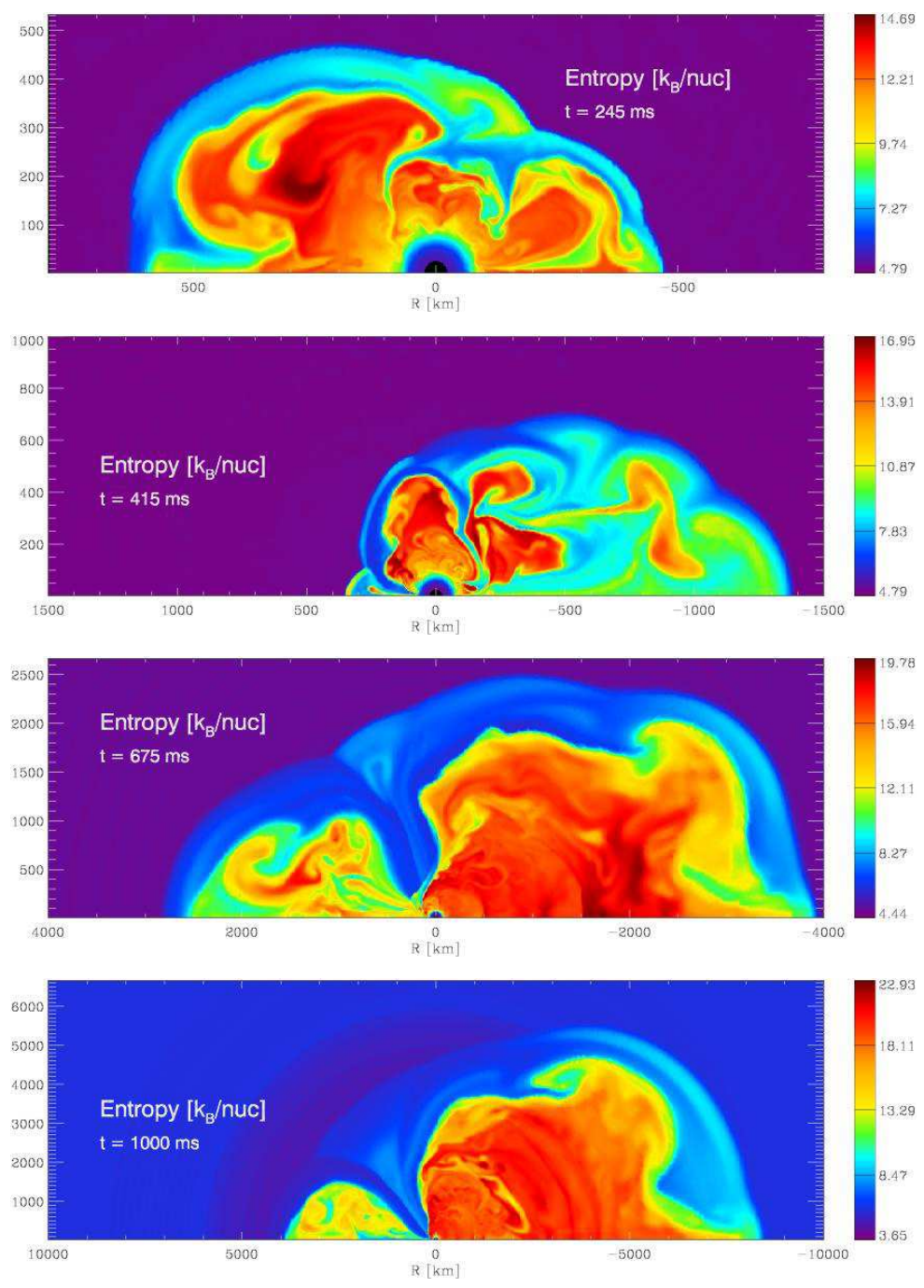

Figure 2. Explosion that is driven by neutrino-energy deposition in combination with convective overturn in the region behind the supernova shock. The anisotropy of the neutrino- and shock-heated ejecta is growing in time and becomes very large due to an increasing contribution of the $m=0, l=1$ mode in the convective pattern. The snapshots (from top to bottom) show the entropy distribution (values between about 4 and $23 k_{\mathrm{B}}$ per nucleon) at post-bounce times $t_{\mathrm{pb}}=245 \mathrm{~ms}, 415 \mathrm{~ms}, 675 \mathrm{~ms}$, and $1000 \mathrm{~ms}$. Note that the radial scales of the figures differ. The neutron star is at the origin of the axially symmetric (2D) grid and plays the role of an isotropic neutrino "light bulb" (Plewa et al., in preparation). 
The high space velocities of young pulsars with a mean around $400 \mathrm{~km} / \mathrm{s}$ (Lyne \& Lorimer 1994; Cordes \& Chernoff 1998) have also been used to argue for an intrinsically asymmetric explosion mechanism. It is very likely that the neutron stars obtained their recoil at the onset of the explosion or shortly afterwards (for a review, see Lai 1991; Lai, Chernoff, \& Cordes 2001). But it is not clear whether the mechanism and the anisotropies of the explosion are directly connected with the pulsar kicks. Also a possible "jet" and perhaps a "counter jet" in the gaseous filaments of the Cas A supernova remnant may neither be linked to the process that started the supernova explosion nor to the runaway velocity of the compact remnant. The latter could have been accelerated (maybe by anisotropic neutrino emission) after the explosion was launched. The jet-like features - if at all caused by processes at the supernova center and not by local inhomogeneities at the forward shock - may have been produced even later, for example by bipolar outflow from a neutron star or a black hole that has accreted from a disk of fallback matter. Interestingly, inspecting the images given by Gotthelf et al. (2001), the dislocation of the compact source relative to the geometrical center of the remnant appears not to coincide with the direction of the possible "jets" (compare also Thorstensen, Fesen, \& van den Bergh 2001).

Rotation plus magnetic fields were proposed as the "most obvious" way to break the spherical symmetry and to explain the global asphericity of core-collapse supernovae (Wheeler, Meier, \& Wilson 2002; Akiyama et al. 2002; Wheeler 2002). It was argued that current numerical calculations may be missing a major ingredient necessary to yield explosions. A proper treatment of rotation and magnetic fields may be necessary to fully understand when and how stellar collapse leads to an explosion. Of course, this might be true. But a confirmation or rejection will require computer models with ultimately the full physics.

It must be stressed, however, that current observations do not necessitate such conclusions and hydrodynamical simulations suggest other possible explanations. Strong convection in the neutrino-heating region behind the supernova shock can account for huge anisotopies of the inner supernova ejecta, even without invoking rotation. If the explosion occurs quickly, much power remains on smaller scales until the expansion sets in and the convective pattern gets frozen in. If, in contrast, the shock radius grows only very slowly and the explosion is delayed for several $100 \mathrm{~ms}$ after bounce, the convective flow can merge to increasingly larger structures. In two-dimensional (2D) hydrodynamic calculations including cooling and heating by neutrinos between the neutron star and the shock (with parameter choices for a central, isotropic neutrino "light bulb" which enabled explosions), we (Plewa et al., in preparation) found 
situations where the convective pattern revealed a contribution of the $l=1, m=0$ mode that was growing with time and was even dominant at about one second after bounce (Fig. 2). Herant (1995) already speculated about such a possibility. Certainly three-dimensional (3D) calculations of the full sphere (and without the coordinate singularity on the axis of the spherical grid) are indispensable to convincingly demonstrate the existence of this phenomenon ${ }^{1}$.

\section{Theoretical Possibilities}

The primary energy source for powering supernovae of massive stars is the gravitational binding energy of the newly formed proto-neutron star or proto-black hole (energy from nuclear reactions contributes at a minor level). To initiate and drive the explosion, energy has to be transferred to the stellar matter above the mass cut to be finally converted to kinetic energy of the ejecta. This could be achieved by hydrodynamical shocks, neutrinos, or magnetic fields as mediators. Collimated outflows or jets of relativistic plasma might also play a role for very special circumstances. Depending on the mediator, the conditions for efficient energy transfer, the corresponding timescale, and the tapped energy reservoir are different. A lot of work has been spent in the past 40 years to identify viable supernova mechanisms and to study the involved physics. The space here is not sufficient to review all important papers. We shall therefore only focus on some key contributions without attempting completeness.

\subsection{Hydrodynamical (Prompt) Mechanism}

When the homologously collapsing inner part of the stellar core is abruptly stopped at core bounce by the stiffening of the nuclear equation of state (EoS), a hydrodynamical shock is formed at the boundary to the supersonically infalling outer part (Colgate \& White 1966). In this case the initial energy of the shock is equal to the kinetic energy of the inner core "at the last moment of good homology" (Mönchmeyer 1993):

$$
E_{\mathrm{sh}, \mathrm{i}} \approx 6 \times 10^{51} \frac{M_{\mathrm{ic}}}{\mathrm{M}_{\odot}}\left(\frac{v_{\max }}{4 \times 10^{9} \mathrm{~cm} \mathrm{~s}^{-1}}\right)^{2} \mathrm{erg},
$$

when $M_{\text {ic }}$ is the mass of the homologously collapsing part of the stellar core and $v_{\max }$ its maximum infall velocity. There is general agreement

\footnotetext{
${ }^{1}$ Another interesting possibility was pointed out by Blondin, Mezzacappa, \& DeMarino (2002). Their 2D (and 3D; Mezzacappa et al. 2002) calculations revealed hydrodynamical instabilities in the accretion flow behind the stalled shock, which instigate large-scale modes even in the absence of neutrino heating. Whether these calculations are of relevance for the supernova problem has be to seen.
} 
now, supported by a large number of numerical calculations (e.g., Bruenn 1985; Bruenn 1989a; Bruenn 1989b; Myra et al. 1987; Myra \& Bludman 1989; Swesty, Lattimer, \& Myra 1994), that this energy is not sufficient for the shock to reach the surface of the iron core: The energy losses from behind the shock by photodisintegration of nuclei to $\alpha$-particles and free nucleons $\left(\sim 1.5 \times 10^{51}\right.$ erg per $\left.0.1 \mathrm{M}_{\odot}\right)$ as well as neutrino escape after shock breakout through the neutrinosphere (some $10^{51} \mathrm{erg}$ ) are so severe that the shock stalls within milliseconds. Only in case of extremely small iron cores $\left(M \lesssim 1.1 \mathrm{M}_{\odot}\right.$; Baron \& Cooperstein 1990) or an extraordinarily soft nuclear EoS (Baron, Cooperstein, \& Kahana 1985; Baron et al. 1987) is the prompt hydrodynamical shock able to disrupt the star. Nevertheless, the hope that the prompt hydrodynamical bounce-shock mechanism might work for some stars in a mass window around $10 \mathrm{M}_{\odot}$ has not been given up completely (Hillebrandt, Wolff, \& Nomoto 1984; Sumiyoshi et al. 2001).

\subsection{Magnetohydrodynamical (MHD) Mechanism}

Soon after the discovery of pulsars the role of rotation and magnetic fields in the supernova explosion was scrutinized (Ostriker \& Gunn 1971; Bisnovatyi-Kogan 1971). Because of flux conservation a seed field in the stellar core can grow significantly during collapse, although for realistic initial fields not to a strength that magnetic pressure could be dynamically important. But winding of field lines in the case of differential rotation, which is natural after the collapse of a spinning core, can further amplify the toroidal field component. If the magnetic pressure becomes comparable to the thermal pressure, magnetohydrodynamical forces can drive an explosion (Müller \& Hillebrandt 1979), and might accelerate axial jets (LeBlanc \& Wilson 1970; Wheeler, Meier, \& Wilson 2002). Also buoyancy instabilities of highly magnetized matter could produce mass motions (Meier et al. 1976). In this case rotational energy of the spinning proto-neutron star is converted to magnetic energy, a part of which might then end up as kinetic energy of the ejecta. Hence,

$$
E_{\mathrm{ej}} \lesssim 5 \times 10^{52} \frac{M_{\mathrm{ns}}}{1.5 \mathrm{M}_{\odot}}\left(\frac{R_{\mathrm{ns}}}{10 \mathrm{~km}}\right)^{2}\left(\frac{T_{\mathrm{rot}}}{1 \mathrm{~ms}}\right)^{-2} \mathrm{erg},
$$

when $T_{\text {rot }}$ is the spin period of the forming neutron star with mass $M_{\mathrm{ns}}$. The field, however, grows linearly with time and therefore the timescale to reach sufficiently strong fields is long compared to the accretion timescale of the collapsed core, unless unrealistically large initial values are assumed (Meier et al. 1976; Müller \& Hillebrandt 1979). Recently it was suggested that the magnetorotational instability (MRI), 
which was discussed in the context of accretion disks by Balbus \& Hawley (1998), might also be in action in the supernova core (Akiyama et al. 2002). Because of an exponential growth, this would reduce the field amplification timescale drastically and would allow for much smaller initial seed fields, too. It remains to be shown, however, whether the field can grow to a dynamically effective strength in a self-consistent model for reasonable assumptions about the rotation of stellar cores and despite of diverse effects that can lead to field saturation or hinder field growth. Moreover, it is unclear whether axial jets can be initiated by the MRI at the conditions in collapsing stellar cores. Implications for supernova nucleosynthesis are unexplored, but might be problematic if MHD explosions are frequent (Meier et al. 1976). The amplification and transport of magnetic fields in convective regions inside the nascent neutron star (Thompson \& Duncan 1993) and in the neutrino-heating layer behind the supernova shock (Thompson \& Murray 2001) also deserve further attention with respect to the generation of pulsar fields.

\subsection{Neutrino-Heating (Delayed) Mechanism}

While neutrinos drain energy from the shock-heated matter immediately after bounce, the situation changes somewhat later when the density and temperature behind the shock have dropped. High-energy neutrinos, emitted abundantly from the hot accretion layer that covers the nascent neutron star, are absorbed with a small, but finite probability (typically $\sim 10 \%$ ) in the postshock gas. If this energy deposition is large enough, the stalled supernova shock can be revived. Since this can happen on a timescale of hundred or more milliseconds after bounce, the explosion is called "delayed" in contrast to the prompt bounce-shock mechanism (Wilson 1985; Bethe \& Wilson 1985). The idea that the energy transfer to the ejecta is mediated by neutrinos goes back to (Colgate \& White 1966). During core collapse and subsequent contraction, the gravitational binding energy of the forming neutron star is first stored as internal energy (degeneracy energy of leptons and thermal energy of nucleons) and only slowly carried away by neutrinos on the diffusion timescale of several seconds. The kinetic energy required for the supernova explosion is of order $10^{51} \mathrm{erg}$ and hence tiny compared to the huge reservoir of binding energy,

$$
E_{\mathrm{kin}} \ll \frac{3}{5} \frac{G M_{\mathrm{ns}}^{2}}{R_{\mathrm{ns}}} \sim \frac{1}{10} M_{\mathrm{ns}} c^{2} \sim 3 \times 10^{53} \mathrm{erg},
$$

where the second expression results from the first by using $R_{\mathrm{ns}} \sim 3 R_{\mathrm{S}} \equiv$ $6 G M_{\mathrm{ns}} / c^{2}$. But just a smaller fraction (about 10\%) of this energy is 
actually radiated in neutrinos during the first hundreds of milliseconds until the explosion starts.

Neutrino heating behind the shock is essentially unavoidable. The temperature in this region drops roughly like $T \propto r^{-1}$ (close to isentropic conditions), which means that the energy loss rate of the stellar plasma by neutrinos produced in $e^{ \pm}$captures on protons and neutrons falls like $Q_{\nu}^{-} \propto T^{6} \propto r^{-6}$. In contrast, the energy deposition by $\nu_{e}$-absorption on neutrons and $\bar{\nu}_{e}$-absorption on protons decreases much more slowly. The heating rate varies roughly proportional to the neutrino energy density (which scales with the summed luminosity of $\nu_{e}$ plus $\bar{\nu}_{e}, L_{\nu}$, divided by the square of the inverse distance $r$ from the neutrinosphere) and the mean squared energy $\left\langle\epsilon_{\nu}^{2}\right\rangle$ (because of the dependence of the absorption cross section on the neutrino energy $\epsilon_{\nu}$ ), i.e., $Q_{\nu}^{+} \propto L_{\nu}\left\langle\epsilon_{\nu}^{2}\right\rangle / r^{2}$. This means that there must be a radial position, the so-called "gain radius" (Bethe \& Wilson 1985), exterior of which neutrino heating dominates neutrino cooling. The heating rate for matter that is disintegrated to free neutrons and protons can be estimated as

$$
Q_{\nu}^{+} \sim 550 \frac{L_{\nu, 53}\left\langle\epsilon_{\nu, 15}^{2}\right\rangle}{r_{7}^{2}} \frac{\mathrm{MeV}}{\mathrm{s}}
$$

per nucleon for a typical luminosity of $\nu_{e}$ plus $\bar{\nu}_{e}$ of $10^{53} \mathrm{erg} / \mathrm{s}, 15 \mathrm{MeV}$ for the rms neutrino energy, and $r$ measured in units of $10^{7} \mathrm{~cm}$. The explosion energy should scale as

$$
E_{\exp } \sim \frac{\Delta M}{m_{\mathrm{u}}} Q_{\nu}^{+} \Delta t_{\mathrm{h}},
$$

where $\Delta M$ is the neutrino-heated mass, $m_{\mathrm{u}}$ the nucleon mass, and $\Delta t_{\mathrm{h}}$ the timescale of neutrino-energy deposition. The latter can be assumed to be roughly the time until nucleons have absorbed an energy comparable to their binding energy in the gravitational potential of the neutron star, i.e.,

$$
\Delta t_{\mathrm{h}} \sim \frac{G M_{\mathrm{ns}} m_{\mathrm{u}}}{Q_{\nu}^{+} r} \sim 40 \mathrm{~ms}
$$

for $M_{\mathrm{ns}} \approx 1.5 \mathrm{M}_{\odot}$. These arguments suggest that the gradual (in contrast to impulsive) energy deposition by neutrinos might not be responsible for a dominant fraction of the net explosion energy of a supernova. Instead, neutrino heating helps to compensate the gravitational binding and thus triggers the outward expansion of the supernova ejecta. The energy per baryon increases further by ongoing energy transfer from neutrinos to the accelerating matter, and finally reaches its limiting value due to the energy release in recombinations of free nucleons to nuclei. This 
recombination energy may account for a major part of the supernova energy. Numerical simulations and analytic considerations (Janka 2001) suggest a mass $\Delta M$ between a few $10^{-2} \mathrm{M}_{\odot}$ and about $10^{-1} \mathrm{M}_{\odot}$. With a typical energy deposited by neutrino heating being around $5 \mathrm{MeV}$ per nucleon (Janka 2001), the explosion energy could range between some $10^{50} \mathrm{erg}$ and several $10^{51} \mathrm{erg}$, when about $8 \mathrm{MeV}$ are set free for each nucleon that recombines. These numbers naturally match the energies of observed normal supernovae (cf. Sect. 2).

\subsection{Jet-Powered Explosions}

Stars with main sequence masses beyond $20-25 \mathrm{M}_{\odot}$ seem to be associated with much more powerful explosions with energies up to several $10^{52} \mathrm{erg}$ (Sect. 2). Such energies are probably out of reach for the neutrino-heating mechanism as described above. The idea has been coined (Popham, Woosley, \& Fryer 1999; MacFadyen \& Woosley 1999) that the core of such stars collapses to a black hole, which then continues to accrete the infalling matter of the progenitor star ("collapsar" or "failed supernova"; Woosley 1993). Provided that the stellar core has retained enough angular momentum during its pre-collapse evolution (or was spun up by a merger with a binary companion), the accretion will proceed at very high rates through a disk or torus on the timescale of viscous angular momentum transport, which is much longer than the dynamical timescale. Therefore the efficiency of energy release can in principle be much higher than in case of spherical accretion. In terms of the rest-mass energy of the accreted matter one can get $E_{\text {acc }}=\xi M_{\text {acc }} c^{2}$ with maximum values between $\xi=0.057$ for a non-rotating black hole and $\xi=0.42$ for an extreme Kerr hole that accretes from a corotating thin disk (Shapiro \& Teukolsky 1983). Since the mass accretion rates are hypercritical and the densities in the disk correspondingly high, photons cannot escape and the energy is set free in form of neutrinos rather than electromagnetic radiation (Popham, Woosley, \& Fryer 1999). The efficiency may be reduced compared to the quoted maximum values if the density and temperature are too low for rapid neutrino production or the disk is very dense and thus becomes nontransparent to neutrinos. In these cases the energy is advected into the black hole by the accretion flow instead of being carried away by neutrinos (Di Matteo, Perna, \& Narayan 2002).

Alternatively, magnetohydrodynamical processes may drive outflows from the disk (e.g., Meier, Koide, \& Uchida 2001; Daigne \& Mochkovitch 2002; Drenkhahn 2002; Drenkhahn \& Spruit 2002). It is also possible that the rotational energy of the spinning black hole is extracted via the 
Blandford and Znajek mechanism (Blandford \& Znajek 1977) through magnetic fields that are anchored in the black hole and couple it to the surrounding disk.

By the annihilation of neutrinos and antineutrinos to $e^{ \pm}$-pairs (Woosley 1993) or the mentioned MHD processes, polar jets could be launched to propagate through the star along the rotation axis (MacFadyen \& Woosley 1999; Aloy et al. 2000; Zhang, Woosley, \& MacFadyen 2002). In case the jets remain collimated and highly relativistic until they break out of the stellar surface (which requires that the star does not possess an extended hydrogen envelope; Matzner 2002) gamma-ray bursts (GRBs) may be triggered. Nonrelativistic jets that expand laterally and sweep up the surrounding progenitor gas, or energetic winds from the accretion torus might be the driving force behind enormously powerful stellar explosions (MacFadyen, Woosley, \& Heger 2001) which are observed as so-called "hypernovae" and GRB-supernovae (Iwamoto et al. 1998). For an overview and more information, see Woosley, Zhang, \& Heger (2002).

The different mechanisms for massive star explosions as described above require different properties of the progenitor stars and different physical conditions in the collapsed stellar cores. The latter are incompletely known, but some of the requirements are more likely fulfilled than others, some combinations of necessary conditions may be more common and more typical, while others might be realized only in rare cases and for very special, exceptional circumstances. It is possible, if not probable, that the diversity of the observed explosions of massive stars and the large number of associated phenomena mean that more than one mechanism is at work. It may also be, however, that the majority of ordinary supernovae can be ascribed to the same central process which might then depend extremely sensitively on yet to be determined parameters.

\section{Do Neutrino-Driven Explosions Work?}

The neutrino-driven mechanism (Wilson 1985; Bethe \& Wilson 1985) involves a minimum of controversial assumptions and uncertain degrees of freedom in the physics of collapsing stars. It relies on the importance of neutrinos and their energetic dominance in the supernova core. After the detection of neutrinos in connection with Supernova 1987A and the overall confirmation of theoretical expectations for the neutrino emission, this is not a speculation any more but an established fact. Of course, this does not mean that such a minimal input is sufficient to understand the cause of supernova explosions and to explain all observable properties of supernovae. But at least it can be taken as a good reason 
to investigate how far one can advance with a minimum of imponderabilities. Detailed numerical simulations are needed to answer the crucial question in this context: Is neutrino-energy deposition efficient enough to drive the explosion?

Spherically symmetric simulations with the current input physics (neutrino interactions and the equation of state of dense matter) do not yield explosions by the neutrino-heating mechanism. There is no controversy about that. All computations are in agreement, independent of Newtonian or relativistic gravity and independent of the neutrino transport being treated in an approximate way by flux-limited diffusion methods (e.g., Myra et al. 1987; Myra \& Bludman 1989; Bruenn 1993; Bruenn, De Nisco, \& Mezzacappa 2001) or very elaborately by solving the frequencyand angle-dependent Boltzmann transport equation (Rampp \& Janka 2000; Mezzacappa et al. 2001; Liebendörfer et al. 2001; Liebendörfer et al. 2002).

Whether neutrinos succeed in reviving the stalled shock depends on the efficiency of the energy transfer to the postshock layer, which in turn increases with the neutrino luminosity and the hardness of the neutrino spectrum. Wilson and collaborators (Wilson \& Mayle 1988; Wilson \& Mayle 1993; Mayle, Tavani, \& Wilson 1993; Totani et al. 1998) have obtained explosions in one-dimensional (1D) simulations for more than ten years now. In these models it is, however, assumed that neutronfinger convection in the hot neutron star boosts the neutrino luminosities. Moreover, a special equation of state with a high abundance of pions in the nuclear matter was used (Mayle, Tavani, \& Wilson 1993), which again leads to higher neutrino fluxes from the neutron star and thus to enhanced energy-deposition behind the shock. Both assumptions are not generally accepted.

Two-dimensional (Herant, Benz, \& Colgate 1992; Herant et al. 1994; Shimizu, Yamada, \& Sato 1994; Burrows, Hayes, \& Fryxell 1995; Janka \& Müller 1996; Mezzacappa et al. 1998; Shimizu et al. 2001) and 3D simulations (Shimizu, Yamada, \& Sato 1993; Fryer \& Warren 2002) as well as theoretical considerations (Bethe 1990) have shown that the neutrinoheating layer is unstable to convective overturn. The associated effects support shock revival and can lead to explosions even in cases where spherical models fail. In the multi-dimensional situation accretion and expansion can occur simultaneously. Downflows of cooler, low-entropy matter that has fallen through the shock, coexist with rising bubbles of high-entropy, neutrino-heated gas (Fig. 2). On the one hand, the downflows carry cool material close to the gain radius where it absorbs energy readily from the intense neutrino fluxes. On the other hand, heated matter rises in bubbles and can expand and cool quickly, which 
reduces the energy loss by the reemission of neutrinos. This also increases the postshock pressure and hence pushes the shock farther out. The gain layer grows and thus more gas can accumulate in the neutrinoheating region. The gas also stays longer in the gain layer, in contrast to one-dimensional models where the matter behind the accretion shock has negative velocity and is quickly advected down to the cooling layer. When the gas arrives there, neutrino emission sets in and extracts again the energy which had been absorbed from neutrino heating shortly before. Due to the combination of all these effects postshock convection enhances the efficiency of the neutrino-heating mechanism. Therefore the multi-dimensional situation is generically different from the spherically symmetric case.

Nevertheless, the existence of convective overturn in the neutrinoheating layer does not guarantee explosions (Janka \& Müller 1996; Mezzacappa et al. 1998). For insufficient neutrino heating the threshold to an explosion will not be overcome. Since neutrinos play a crucial role, an accurate description of the neutrino physics - transport and neutrinomatter interactions - is indispensable to obtain conclusive results about the viability of the neutrino-driven mechanism. All previously published multi-dimensional explosion models, however, have employed some crude approximations or simplifications in the treatment of neutrinos.

\section{A New Generation of Multi-Dimensional Supernova Simulations}

In order to take a next step of improvement in supernova modelling, we have coupled a new Boltzmann solver for the neutrino transport to the PROMETHEUS hydrodynamics code. The combined program is called MuDBaTH (Multi-Dimensional Boltzmann Transport and Hydrodynamics) and allows for spherically symmetric as well as multidimensional simulations (Rampp \& Janka 2002). Below we present some results of $1 \mathrm{D}$ and our first $2 \mathrm{D}$ supernova simulations with this new code.

\subsection{Technical Aspects and Input Physics}

For the integration of the equations of hydrodynamics we employ the Newtonian finite-volume code PROMETHEUS (Fryxell, Müller, \& Arnett 1989), which was supplemented by additional problem specific features (Keil 1997). PROMETHEUS is a direct Eulerian, time-explicit implementation of the Piecewise Parabolic Method (PPM) of Colella \& Woodward (1994). As a second-order Godunov scheme employing a Riemann solver it is particularly well suited for following discontinuities in the fluid flow like shocks or boundaries between layers of different 
chemical composition. A notable advantage in the present context is its capability of tackling multi-dimensional problems with high computational efficiency and numerical accuracy. Our code makes use of the "Consistent Multifluid Advection (CMA)" method (Plewa \& Müller 1999) for ensuring accurate advection of different chemical components in the fluid, and switches from the original PPM method to the more diffusive HLLE solver of Einfeldt (1988) in the vicinity of strong shocks to avoid spurious oscillations (the so-called "odd-even decoupling" phenomenon) when such shocks are aligned with one of the coordinate lines in multidimensional simulations (Quirk 1994; Kifonidis 2000; Plewa \& Müller 2001).

The Boltzmann solver scheme is described in much detail elsewhere (Rampp \& Janka 2002). The integro-differential character of the Boltzmann equation is tamed by applying a variable Eddington factor closure to the neutrino energy and momentum equations (and the simultaneously integrated first and second order moment equations for neutrino number). For this purpose the variable Eddington factor is determined from the solution of the Boltzmann equation, and the system of Boltzmann equation and its moment equations is iterated until convergence is achieved. Employing this scheme in multi-dimensional simulations in spherical coordinates, we solve the radius- (and energy-) dependent moment equations on the different angular bins of the numerical grid but calculate the variable Eddington factor only once on an angularly averaged stellar background. This approximation is good only for situations without significant global deformations. Since the iteration of the Boltzmann equation has to be done only once per time step, appreciable amounts of computer time can be saved (Rampp \& Janka 2002). We point out here that it turned out to be necessary to go an important step beyond this simple "ray-by-ray" approach. Physical constraints, namely the conservation of lepton number and entropy within adiabatically moving fluid elements, and numerical requirements, i.e., the stability of regions which should not develop convection according to a mechanical stability analysis, make it necessary to take into account the coupling of neighbouring rays at least by lateral advection terms and neutrino pressure gradients (Buras et al., in preparation).

General relativistic effects are treated only approximately in our code (Rampp \& Janka 2002). The current version contains a modification of the gravitational potential by including correction terms due to pressure and energy of the stellar medium and neutrinos, which are deduced from a comparison of the Newtonian and relativistic equations of motion. The neutrino transport contains gravitational redshift and time dilation, but ignores the distinction between coordinate radius and proper radius. 
This simplification is necessary for coupling the transport code to our basically Newtonian hydrodynamics. Although a fully relativistic treatment would be preferable, tests showed that these approximations seem to work satisfactorily well (Liebendörfer et al., in preparation), at least as long as there are only moderate $(\sim 10-20 \%)$ deviations of the metric coefficients from unity and the infall velocities do not reach more than $10-20 \%$ of the speed of light in decisive phases of the evolution.

As for the neutrino-matter interactions, we discriminate between two different sets of input physics. On the one hand we have calculated models with conventional ("standard") neutrino opacities, i.e., a description of the neutrino interactions which follows closely the one used by Bruenn and Mezzacappa and collaborators (Bruenn 1985; Mezzacappa \& Bruenn 1993a; Mezzacappa \& Bruenn 1993b). It assumes nucleons to be uncorrelated, infinitely massive scattering targets for neutrinos. In these reference runs we have usually also added neutrino pair creation and annihilation by nucleon-nucleon bremsstrahlung (Hannestad \& Raffelt 1998). Details of our implementation of these neutrino processes can be found elsewhere (Rampp \& Janka 2002).

A second set of models was computed with an improved description of neutrino-matter interactions. Besides including nucleon thermal motions and recoil, which means a detailed treatment of the reaction kinematics and allows for an accurate evaluation of nucleon phase-space blocking effects, we take into account nucleon-nucleon correlations (following Burrows \& Sawyer 1998; Burrows \& Sawyer 1999), the reduction of the nucleon effective mass, and the possible quenching of the axial-vector coupling in nuclear matter (Carter \& Prakash 2002). In addition, we have implemented weak-magnetism corrections as described by Horowitz (2002). The sample of neutrino processes was enlarged by also including scatterings of muon and tau neutrinos and antineutrinos off electron neutrinos and antineutrinos and pair annihilation reactions between neutrinos of different flavors [i.e., $\nu_{\mu, \tau}+\bar{\nu}_{\mu, \tau} \longleftrightarrow \nu_{e}+\bar{\nu}_{e}$; Buras et al. (2002)].

Our current supernova models are calculated with the nuclear equation of state of Lattimer \& Swesty (1991) using an incompressibility modulus of $K=180 \mathrm{MeV}$ (other values of the incompressibility of bulk nuclear matter in the Lattimer \& Swesty EoS cause only minor differences, see Thompson, Burrows, \& Pinto 2002). For the density regime below $6 \times 10^{7} \mathrm{~g} / \mathrm{cm}^{3}$ we switch to an equation of state that considers electrons, positrons and photons, and nucleons and nuclei with an approximative treatment for composition changes due to nuclear burning and shifts of nuclear statistical equilibrium (Rampp \& Janka 2002). 
Table 1. Input physics for our set of computed models. See Sect. 5.1 for details.

\begin{tabular}{|c|c|c|c|c|c|c|}
\hline Model & Dim. & Gravity & $\nu$ Reactions & Transport & $W e d g e^{a}$ & Zones \\
\hline s15Nso_1d.a & $1 \mathrm{D}$ & Newtonian & standard & Case A & & \\
\hline s15Nso_1d.b & $1 \mathrm{D}$ & Newtonian & standard & Case B & & \\
\hline s15Nso_2d.a & $2 \mathrm{D}$ & Newtonian & standard & Case A & $\pm 27^{\circ}$ & 20 \\
\hline s15Gso_1d.b & $1 \mathrm{D}$ & approx. GR & standard & Case B & & \\
\hline s15Gso_1d.b* & $1 \mathrm{D}$ & approx. GR & standard $^{b}$ & Case B & & \\
\hline s15Gio_1d.a & $1 \mathrm{D}$ & approx. GR & improved $^{c}$ & Case A & & \\
\hline s15Gio_2d.a & $2 \mathrm{D}$ & approx. GR & improved & Case A & $\pm 43.2^{\circ}$ & 32 \\
\hline s15Gio_1d.b & $1 \mathrm{D}$ & approx. GR & improved & Case B & & \\
\hline s15Gio_2d.b & $2 \mathrm{D}$ & approx. GR & improved & Case B & $\pm 43.2^{\circ}$ & 32 \\
\hline
\end{tabular}

${ }^{a}$ Angular wedge of the spherical coordinate grid around the equatorial plane.

${ }^{b}$ Calculation without neutrino-pair creation by nucleon-nucleon bremsstrahlung.

${ }^{c}$ Calculation without the neutrino-antineutrino processes of Buras et al. (2002).

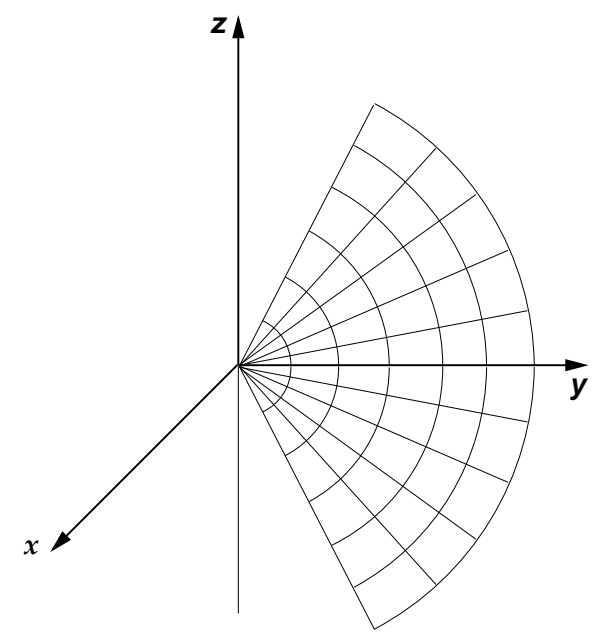

Figure 3. Computational grid for the $2 \mathrm{D}$ simulations. The wedge is placed around the equatorial plane of the spherical coordinate grid. Azimuthal symmetry around the $z$-axis is assumed, and periodic conditions are used at the lateral grid boundaries.

\subsection{Models and Results}

We have performed a number of core-collapse simulations in spherical symmetry and then followed the post-bounce evolution in one and two dimensions. All described calculations were started from a $15 \mathrm{M}_{\odot}$ progenitor star, Model s15s7b2, provided to us by S. Woosley. Adopting the naming, we label our models by s15N for Newtonian runs and s15G for runs with approximate treatment of general relativity, followed by letters "so" when "standard neutrino opacities" were used and by "io" in case of our state-of-the-art improvement of the description of neutrinomatter interactions. The model names have a suffix that discriminates between 1D ("_1d") and 2D simulations ("_2d"). For the 2D models we 

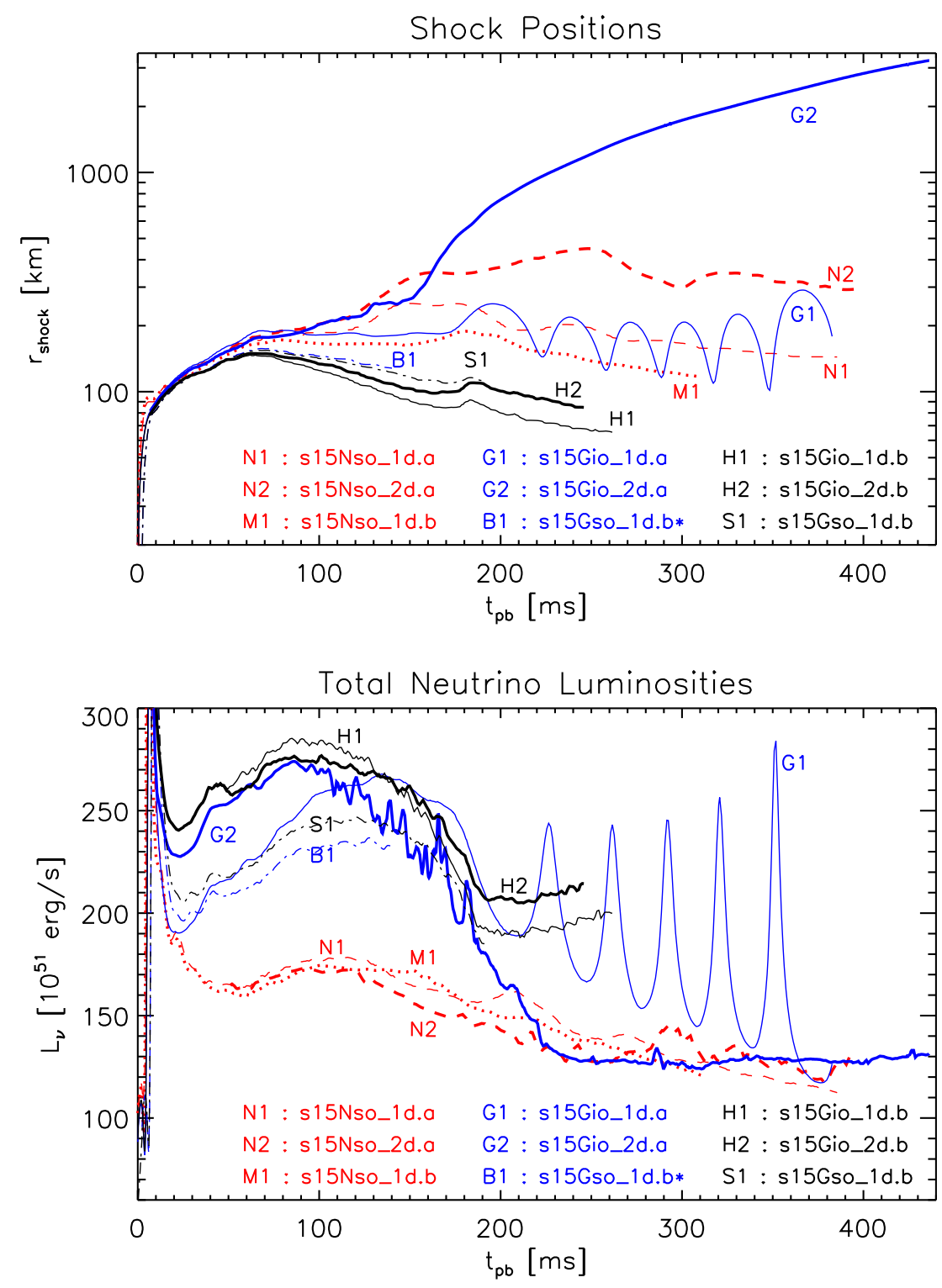

Figure 4. Shock trajectories (top) and total neutrino luminosities (sum of luminosities of $\nu_{e}, \bar{\nu}_{e}$, and muon and tau neutrinos and antineutrinos; bottom) for all models. Bold lines correspond to two-dimensional simulations. Model H2 (s15Gio_2d.b) is the most complete $2 \mathrm{D}$ simulation. It was computed with the improved set of neutrino opacities, relativistic effects, and all velocity-dependent terms retained in the neutrino transport equations. 

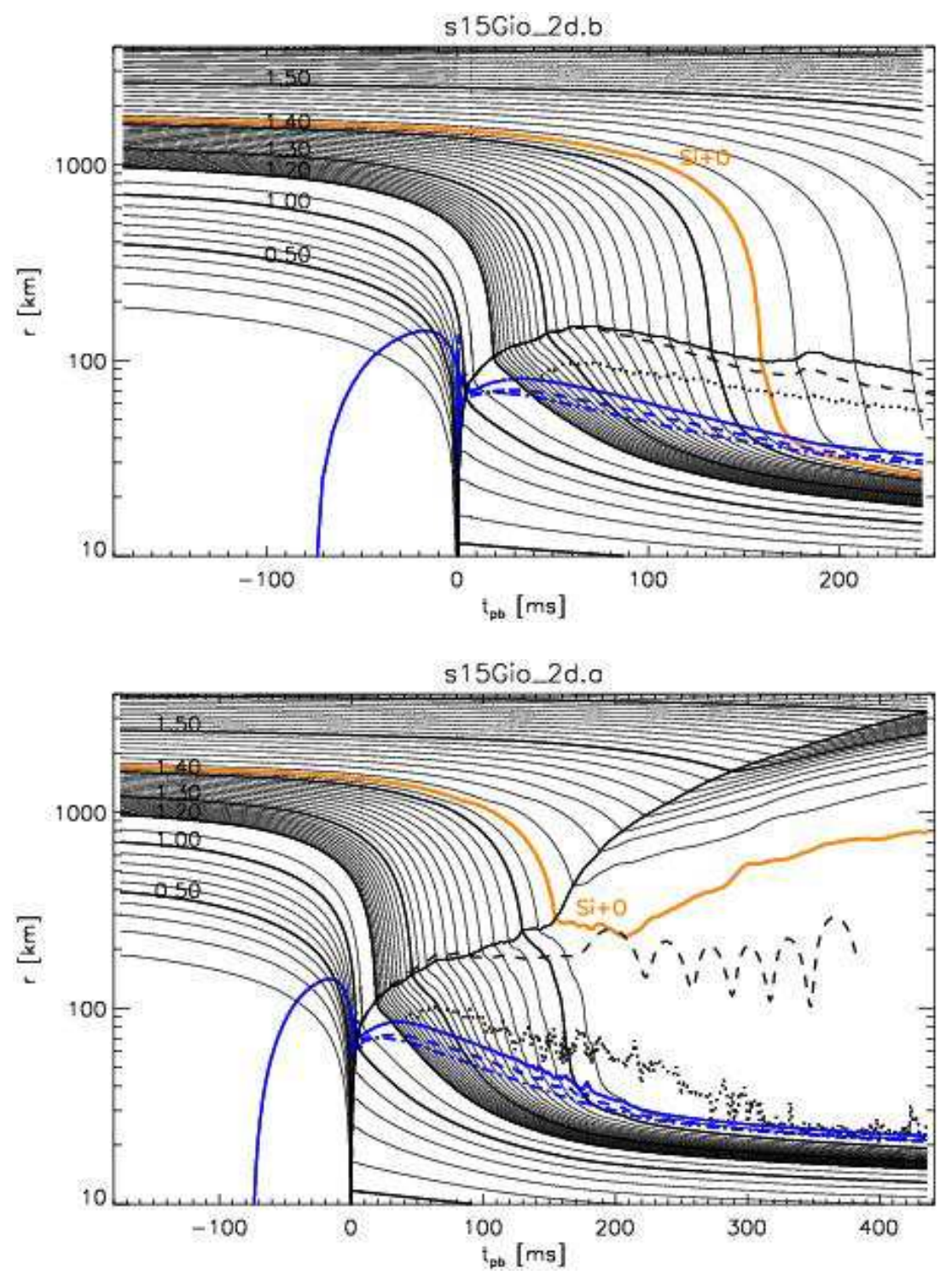

Figure 5. Trajectories of mass shells (time being normalized to bounce) for the non-exploding (top) and the exploding 2D model. In the latter case one can see the shock starting a rapid expansion at about $150 \mathrm{~ms}$ after bounce. The dashed lines indicate the shock positions in the corresponding 1D simulations, where no explosions were obtained. The angle-averaged gain radius is given by the dotted line, and the neutrinospheres of $\nu_{e}, \bar{\nu}_{e}$ and heavy-lepton neutrinos (lower solid, dashed, and dashdotted lines, respectively) are also marked. 
used a spherical coordinate grid with 20 or 32 equidistant zones within an angular wedge from $-27^{\circ}$ to $+27^{\circ}$ or from $-43.2^{\circ}$ to $+43.2^{\circ}$, respectively, around the equatorial plane and assumed periodic conditions at the boundaries (Fig. 3).

We have varied yet another aspect in our simulations. Some of the models were computed with a version of the transport code where the velocity dependent (Doppler shift and aberration) terms in the neutrino momentum equation (and the corresponding terms in the Boltzmann equation for the antisymmetric average of the specific intensity; see Rampp \& Janka 2002) were omitted. These terms are formally of order $v / c$ and should be small for low velocities. This simplification of the neutrino transport, our so-called "Case A", was used in the models which have names ending with the letter "a". Models with neutrino transport including all velocity dependent terms also in the neutrino momentum equation ("Case B") can be identified by the ending "b" of their names. Table 1 provides an overview of the computed models.

The shock trajectories and corresponding total neutrino luminosities of all models are displayed in Fig. 4. No explosions, neither with the standard nor with the improved description of the neutrino opacities, were obtained with the most complete implementation of the transport equations, Case B (see lines labeled with M1, H1, H2, S1, B1, corresponding to Models s15Nso_1d.b, s15Gio_1d.b, s15Gio_2d.b, s15Gso_1d.b and s15Gso_1d.b*, respectively). The shock trajectories of this sample of models form a cluster that is clearly separated from the models computed with the transport version of Case A, which generally show a larger shock radius and therefore more optimistic conditions for explosions.

The simplification of the neutrino transport in Case A has a remarkable consequence which is obvious from a comparison of the $2 \mathrm{D}$ runs of Models s15Gio_2d.a and s15Gio_2d.b, which both were performed with our approximation of relativistic effects and the state-of-the-art improvement of neutrino-matter interactions (cf. Sect. 5.1). While Model s15Gio_2d.b fails to explode, the stalled shock in Model s15Gio_2d.a is successfully revived by neutrino heating because very strong convection can develop in the gain region. The time evolution of both models is displayed by the trajectories of mass shells in Fig. 5 .

The reason for this dramatic difference can be understood in the following way. Some of the velocity dependent terms (those in which derivatives with respect to the neutrino energy do not show up) in the neutrino momentum equation have a simple formal interpretation: In regions with mass infall (negative velocity) they effectively act like a reduction of the neutrino-medium interaction on the right hand side of this equation. The corresponding changes can be $10 \%$ or more for neutrino 
energies in the peak of the spectrum, depending also on time, radius, and the size of the postshock velocities. As a consequence of the omission of these terms in Model s15Gio_2d.a, neutrinos are impeded in their streaming. Therefore the comoving-frame neutrino (energy) density is increased (see Fig. 6). This is associated with slightly lower neutrino losses in the cooling layer around the neutrinosphere and a significantly enhanced neutrino heating between gain radius and shock.

Conversely, the inclusion of the velocity-dependent terms in the neutrino momentum equation has negative consequences for the shock propagation in Model s15Gio_2d.b. Although the differences at early postbounce times are moderate (10-30\%, depending on the quantity) the accumulating effects during the first $80 \mathrm{~ms}$ after bounce clearly damp the shock expansion and finally lead to a dramatic shock recession after the initial phase of expansion. Before this happens postshock convection has not become strong enough to change the evolution. With the onset of contraction, the postshock velocities decrease (become more negative) quickly, neutrino-heated matter is rapidly advected down through the gain radius and loses its energy by the reemission of neutrinos. The larger negative values of the postshock velocity enhance the influence of the velocity-dependent terms and reduce the heating efficiency even more. Because of this disastrous feedback, the gain region shrinks to a very narrow layer, a fact which suppresses the convective activity lateron. This is demonstrated by Figs. 7 and 8, which show that the convection is weak in Model s15Gio_2d.b but very strong in Model s15Gio_2d.a.

Due to a combination of unfavorable effects and a continuously amplifying negative trend, Model s15Gio_2d.b remains below the explosion threshold while Model s15Gio_2d.a is just above that critical limit. In the vicinity of the threshold the long-time evolution of the collapsing stellar core therefore depends very sensitively on "smaller details" of the neutrino transport.

The two simulations also demonstrate that a sufficiently large shock radius for a sufficiently long time is crucial for the growth of convective overturn in the neutrino-heating layer. Only when convection behind the shock becomes strong it can be decisive for getting an explosion. This latter fact is known from previous multi-dimensional simulations (Janka \& Müller 1996; Mezzacappa et al. 1998) and is again confirmed by a comparison of Model s15Gio_2d.a with the corresponding one-dimensional simulation, Model s15Gio_1d.a. It is interesting that the Newtonian 2D run, Model s15Nso_2d.a (with standard opacities) does marginally fail, whereas Model s15Gio_2d.a with relativistic corrections (and stateof-the-art neutrino reactions) succeeds. The influence of the neutrino opacities can be directly seen by comparing Models s15Gso_1d.b and 

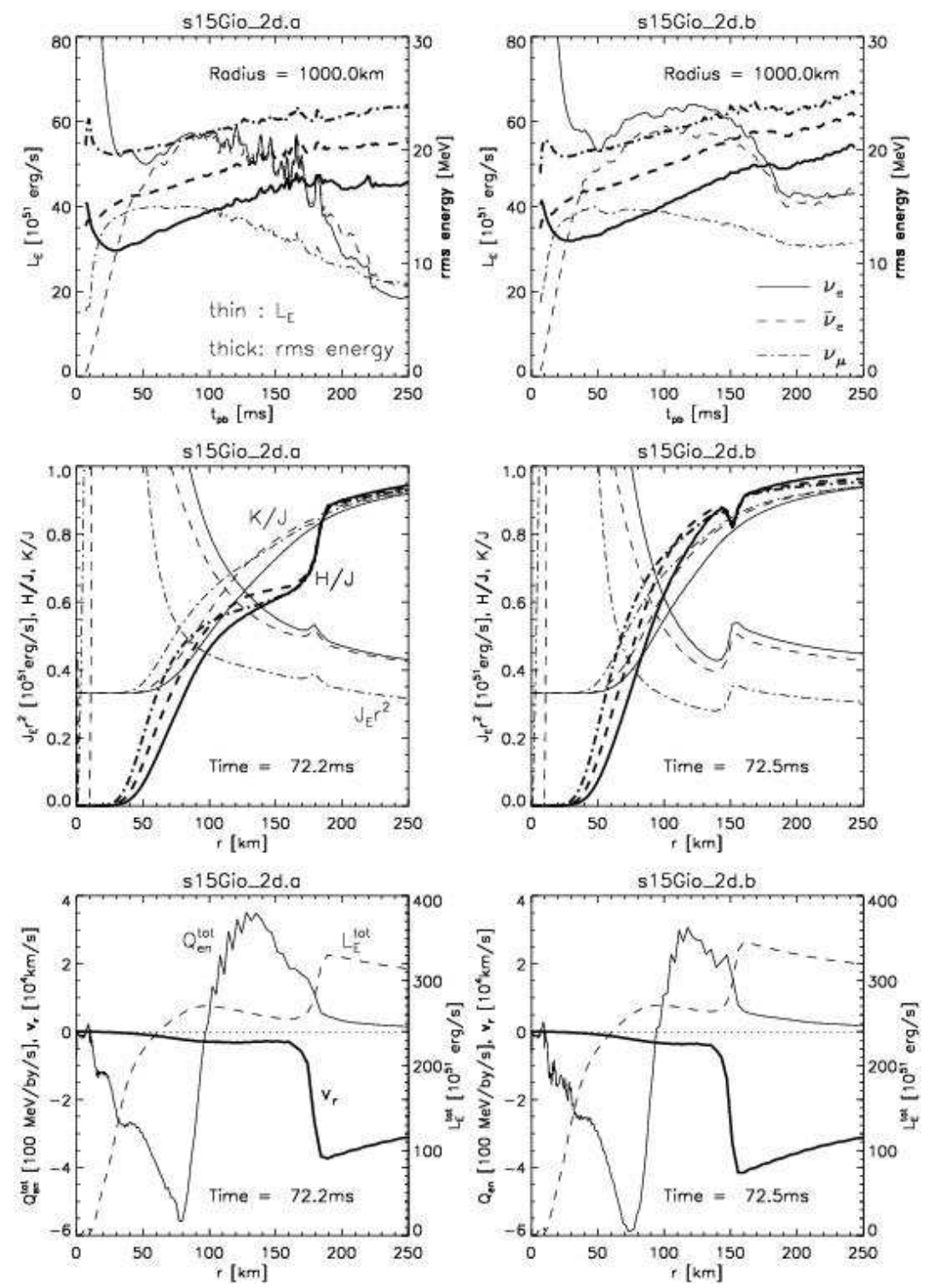

Figure 6. Comparison of the exploding 2D model (Model s15Gio_2d.a; left) and the non-exploding 2D model (Model s15Gio_2d.b; right). The top panels show the (comoving-frame) neutrino luminosities and rms energies as functions of time after bounce. The middle panels display the neutrino energy moment $J$, multiplied by $r^{2}$ and the flux factor $H / J$ and Eddington factor $K / J$ for both models at post-bounce time $t=72.5 \mathrm{~ms}$, which corresponds roughly to the moment of maximum shock expansion in Model s15Gio_2d.b (cf. Fig. 5). The lower panels give the total neutrino luminosities and neutrino heating rates in the comoving frame of the stellar fluid at the same time. 


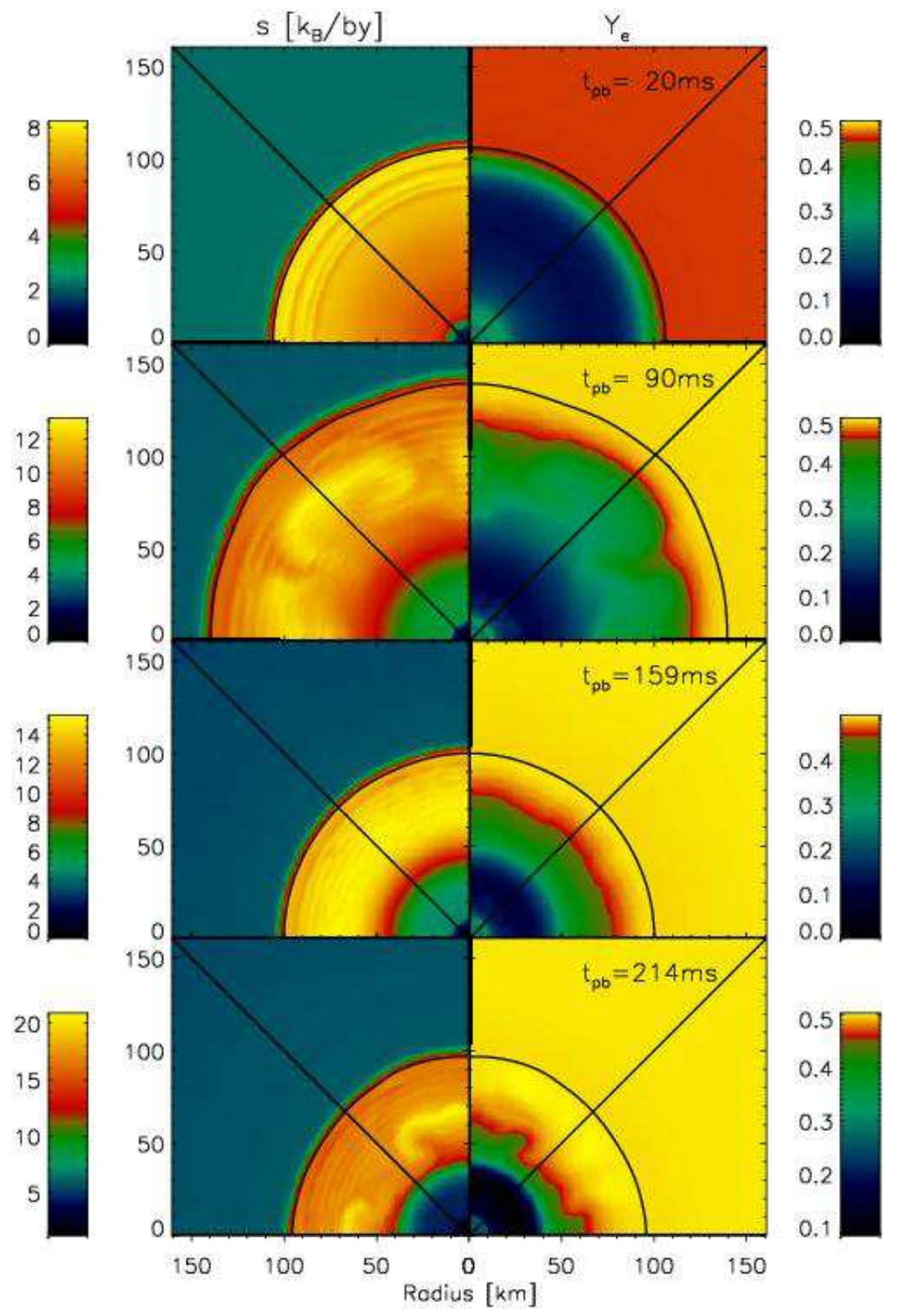

Figure \%. Convection in the neutrino-heating region for the non-exploding 2D model (Model s15Gio_2d.b) at the post-bounce times indicated in the plots. The figures show the entropy distribution (left) and the electron fraction (proton-to-baryon ratio). A wedge of $\pm 43.2^{\circ}$ around the equatorial plane (marked by the diagonal solid lines) of the spherical coordinate grid was used for the computation. 


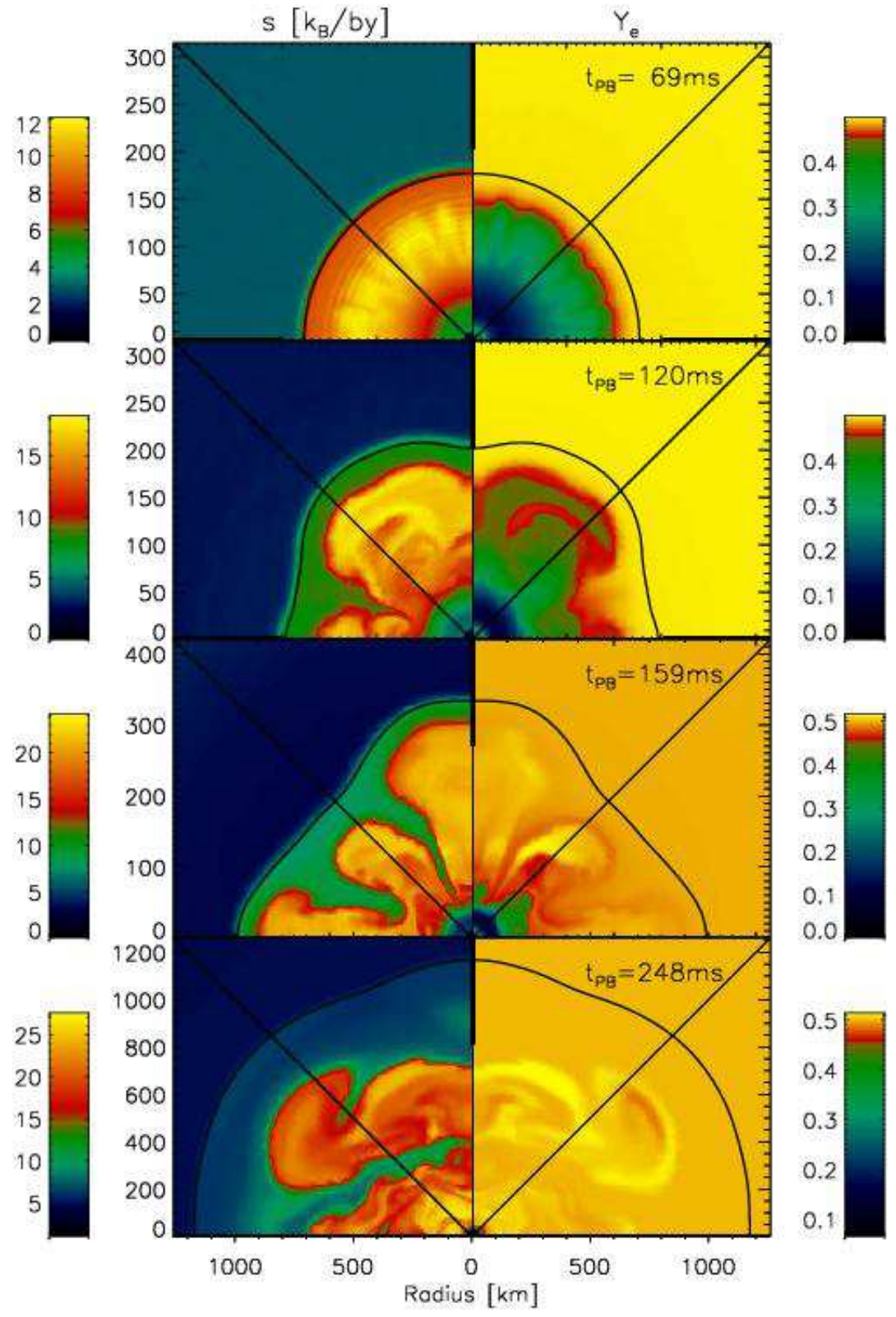

Figure 8. Same as Fig. 7, but for the exploding 2D model, Model s15Gio_2d.a. Note the different radial scales of the four snapshots. 
s15Gio_1d.b. It is not dramatic, but sufficient to justify the inclusion of the improvements described in Sect. 5.1. Neutrino-pair creation by bremsstrahlung makes a minor difference during the considered phases of the evolution (Model s15Gso_1d.b* vs. Model s15Gso_1d.b).

We also found that Ledoux convection sets in below the neutrinosphere already shortly after bounce (Fig. 9). It is persistent until the end of our simulations and slowly digs farther into the star as already found in the simulations of Keil, Janka, \& Müller (1996). But the convective activity is so deep inside the neutron star that its effects on the $\nu_{e}$ and $\bar{\nu}_{e}$ luminosities and on the supernova dynamics are insignificant.

\section{Conclusions and Outlook}

We believe that our 2D models with a Boltzmann solver for the neutrino transport and a state-of-the-art description of neutrino-matter interactions have considerably reduced the uncertainties associated with the treatment of the neutrino physics in previous multi-dimensional simulations. With the most complete implementation of the transport physics we could not obtain explosions. This result suggests that the neutrino-driven mechanism fails with the employed input physics, at least in case of the considered $15 \mathrm{M}_{\odot}$ star. We do not think that the approximate treatment of general relativistic effects is likely to jeopardize this conclusion. A comparison with fully relativistic one-dimensional calculations (Liebendörfer et al., in preparation) is very encouraging. Because of the remarkable similarity of the shock trajectories of different progenitors in spherical symmetry (Liebendörfer et al. 2002), it is probable that our negative conclusion is also valid for other pre-collapse configurations with a similar structure. Significant star-to-star variations of the progenitor properties with a non-monotonic dependence on the stellar mass (Woosley, Heger, \& Weaver 2002), however, suggest that multi-dimensional core-collapse simulations of a larger sample of stars are needed before one can make final, more generally valid statements.

Another concern may be the omission of lateral neutrino fluxes in our multi-dimensional transport approximation. We describe the neutrino losses from the hot spots that appear at locations where the low-entropy downflows plunge into the neutrinospheric layer, by just a radial flux. In reality the neutrinos produced in this semi-transparent matter would be radiated away more isotropically, a fact which could lead to a larger reabsorption probability in the surrounding medium. The corresponding increase of the heating efficiency might not be very large, but could still be relevant because of the close proximity of our models to an explo- 

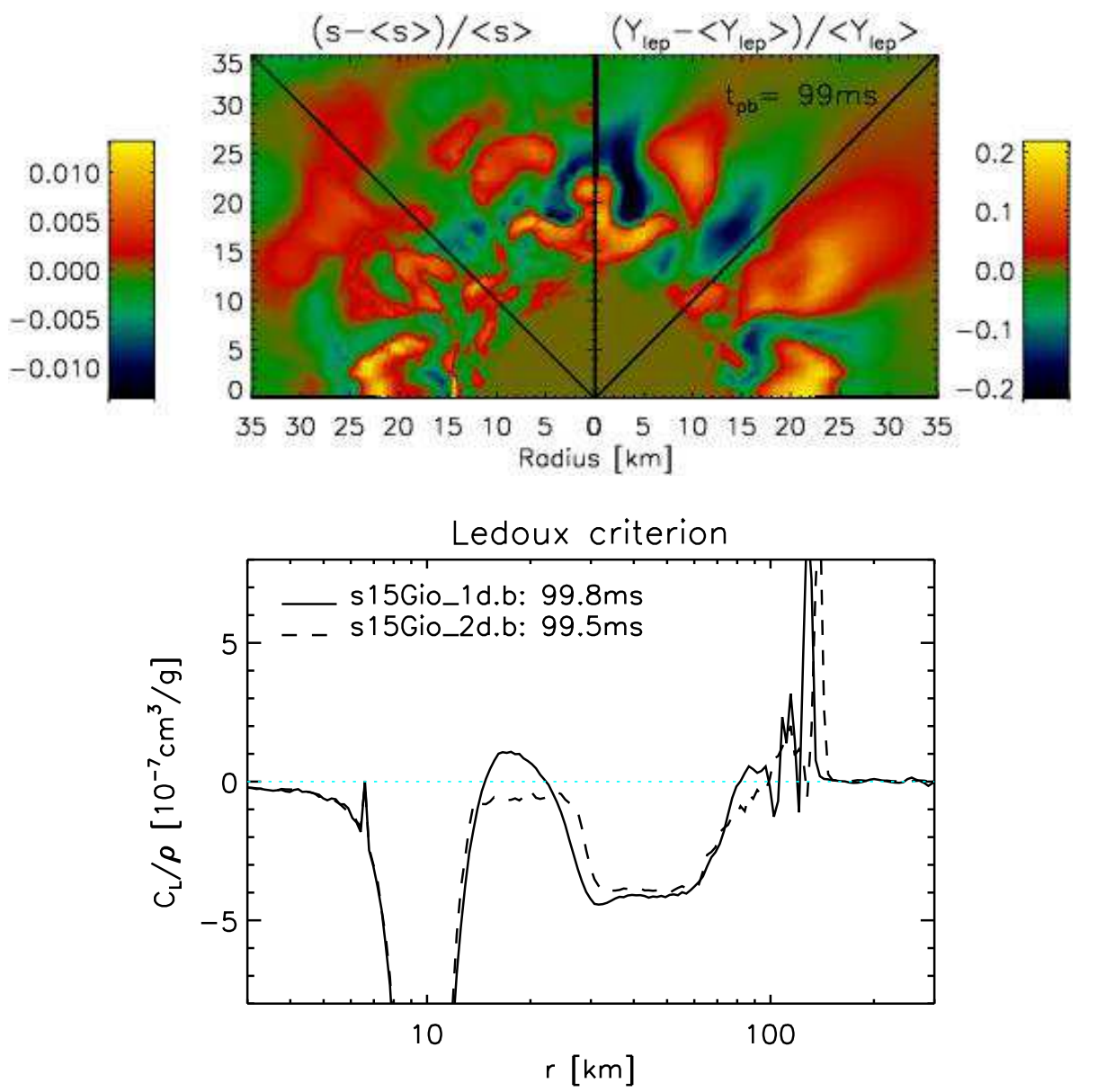

Figure 9. Convection below the neutrinosphere at about $100 \mathrm{~ms}$ after core bounce in Model s15Gio_2d.b. The upper figure shows the relative fluctuations of entropy (left) and lepton fraction (right), normalized to the mean values at a given radius. The lower figure displays the Ledoux criterion for the 1D Model s15Gio_1d.b (without convection; solid line) and the 2D Model s15Gio_2d.b (dashed line). Positive values indicate convectively unstable conditions, once in an inner region (located at about 15-30 km) below the neutrinosphere and another time in the neutrino-heating layer behind the shock.

sion. The supernova problem is highly nonlinear and surprises may lurk behind every corner.

It would therefore be premature to conclude that the neutrino-driven mechanism fails and that not even postshock convection can alter this unquestioned outcome of all current spherical models. Besides studying 


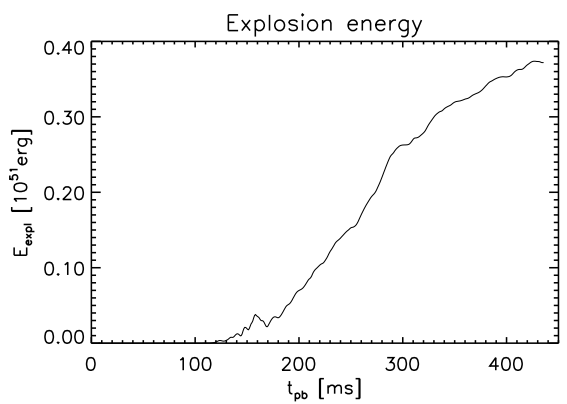

Figure 10. Explosion energy as function of time for Model s15Gio_2d.a.

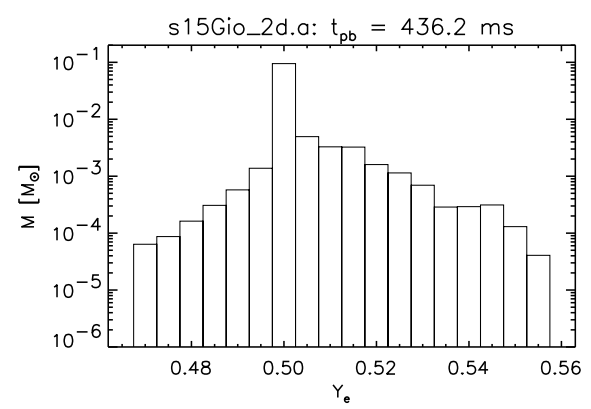

Figure 11. Ejecta mass vs. $Y_{e}$ for Model s15Gio_2d.a at $436 \mathrm{~ms}$ after bounce. The total mass of ${ }^{56} \mathrm{Ni}$ is about $0.1 \mathrm{M}_{\odot}$.

other progenitors with multi-dimensional simulations, one should also investigate the effects of rotation during the neutrino-heating phase and long-time post-bounce evolution of a supernova. Previous simulations found weaker explosions in case of rotating models (Fryer \& Heger 2000). But these calculations were performed with a much simplified treatment of the neutrinos and showed powerful, nearly prompt explosions already in the absence of rotation. It cannot be excluded that even a moderate amount of rotation might have significant consequences when added to nonrotating models which explode only with a long time delay after core bounce or even fail to do so. Naturally, three-dimensional simulations are ultimately desirable and could reveal generic differences compared to $2 \mathrm{D}$, in particular in combination with rotation. The recent results of (Fryer \& Warren 2002), which suggest a large degree of similarity between $2 \mathrm{D}$ and $3 \mathrm{D}$ results, are groundbreaking but again must be interpreted with caution. The serious approximations of the neutrino physics and the rapid development of the explosion in these models do not allow too far-reaching conclusions.

Another major uncertainty of current supernova models is associated with the incompletely known physics in the nuclear and supranuclear medium. Not only does the high-density EoS affect the core bounce, shock formation, and prompt shock propagation, it also determines the internal properties of the hot neutron star and thus its neutrino emission, both indirectly and directly. Hadronic degrees of freedom in addition to nucleons in the supranuclear matter, e.g., hyperons, pions or kaons, can reduce the stiffness of the EoS at very high densities. The possible effects on the supernova explosion are essentially unexplored. A softening of the EoS can, for example, lead to a faster contraction and heating of 
the nascent neutron star. This in turn also determines the density and temperature profile in the vicinity of the neutrinosphere where most of the neutrino flux is produced during the crucial phase of delayed shock revival.

Our successfully exploding 2D model, Model s15Gio_2d.a, at least demonstrates that simulations which include the effects of postshock convection are rather close to an explosion. Therefore modest changes of the neutrino emission and transport (in our case the omission of the velocity-dependent terms in the neutrino-momentum equation) seem to be already sufficient to push them beyond the critical threshold. The properties of the explosion in this case are very encouraging and may support one's belief in the basic viability of the delayed explosion mechanism. At $440 \mathrm{~ms}$ after bounce the shock has arrived at a radius of more than $3000 \mathrm{~km}$ and is expanding with about $10000 \mathrm{~km} / \mathrm{s}$ (Fig. 4). The explosion of this model does not become very energetic (Fig. 10). The energy is just $\sim 4 \times 10^{50} \mathrm{erg}$ at that time and grows only very slowly. This may not be a serious problem if one recalls the large spread of energies of observed supernovae (Supernova 1999br, for example, is estimated to have an ejecta mass of $14 \mathrm{M}_{\odot}$ and an explosion energy of about $6 \times 10^{50} \mathrm{erg}$; Hamuy 2002). Moreover, the explosion energy is a very steep function of the physical conditions in models which are above the explosion threshold (Janka \& Müller 1995). A significant increase of the energy may therefore not require large changes.

Since the explosion starts rather late (at $\sim 150 \mathrm{~ms}$ post bounce), the proto-neutron star has accreted enough matter to have attained an initial baryonic mass of $1.4 \mathrm{M}_{\odot}$. Therefore our simulation does not exhibit the problem of previous successful multi-dimensional calculations which produced neutron stars with masses on the lower side of plausible values $\left(\sim 1.1 \mathrm{M}_{\odot}\right)$. Also another problem of published explosion models (e.g., Herant et al. 1994; Burrows, Hayes, \& Fryxell 1995; Janka \& Müller 1996; Fryer 1999) has disappeared: The ejecta mass with $Y_{e} \lesssim 0.47$ is less than $\sim 10^{-4} \mathrm{M}_{\odot}$ in our calculation (Fig. 11), thus fulfilling a constraint pointed out by Hoffman et al. (1996) for supernovae if they should not overproduce the $N=50$ (closed neutron shell) nuclei, in particular ${ }^{88} \mathrm{Sr},{ }^{89} \mathrm{Y}$ and ${ }^{90} \mathrm{Zr}$, relative to the Galactic abundances. Of course, final statements about explosion energy, ejecta composition, and the neutron star mass (which may change due to later fallback, especially when the explosion energy remains low) require to follow the evolution for a longer time. 


\section{Final Words}

There is still a long way to go until we will have arrived at a "standard model" for massive star explosions. Current numerical simulations are not advanced enough to convincingly and self-consistently demonstrate the viability of any of the explosion scenarios described in Sect. 3. Despite of respectable progress in treating the neutrino transport, general relativity, and multi-dimensional aspects in the most detailed numerical calculations, the models are far from being complete. Neglecting potentially important physics like, e.g., magnetic fields, one cannot be sure that one is searching for the actual cause of massive star explosions at the right place. Conversely, the existing simulations also do not bring us in a position to reject the idea that neutrinos deliver the required energy in the ejecta.

With the lack of direct observational information and being confronted with the fact that our knowledge about the physics in collapsing stellar cores is incomplete, there is no alternative to systematically explore the space of possibilities by hydrodynamical simulations. Since "the output of complex numerical models is as inscrutable as nature herself" (Emanuel 1991), it seems advisable to first develop reliable and accurate supernova models with a minimum of questionable assumptions and then to move on step by step to expanding them towards larger complexity and more completeness.

For the time being, one's favorate choice of the supernova mechanism is more a matter of taste and belief than based on undisputable facts and logical reasoning. The "ifs" and "buts" in current models are still too many. One should be cautious not to put too much money on any single possibility right now.

\section{Acknowledgments}

We are indebted to K. Takahashi for providing us routines to calculate the improved neutrino-nucleon interactions, and to C. Horowitz for correction formulae for the weak magnetism. We also thank M. Liebendörfer for making output data of his simulations available to us for comparisons. The Institute for Nuclear Theory at the University of Washington is acknowledged for its hospitality and the Department of Energy for support during a visit of the Summer Program on Neutron Stars, during which most of the work leading to Fig. 2 was done. HTJ, RB and MR are grateful for support by the Sonderforschungsbereich 375 on "Astroparticle Physics" of the Deutsche Forschungsgemeinschaft. TP was supported in part by the US Department of Energy under Grant No. B341495 to the Center of Astrophysical Thermonuclear Flashes at the University 
of Chicago, and in part by the grant 2.P03D.014.19 from the Polish Committee for Scientific Research. He performed his simulations on the CRAY SV1-1A at the Interdisciplinary Centre for Computational Modelling in Warsaw. The 2D simulations with Boltzmann neutrino transport were only possible because a node of the new IBM "Regatta" supercomputer was dedicated to this project by the Rechenzentrum Garching. Computations were also done on the NEC SX-5/3C of the Rechenzentrum Garching, and on the CRAY T90 and CRAY SV1ex of the John von Neumann Institute for Computing (NIC) in Jülich.

\section{References}

Aloy, M.A., Müller, E., Ibáñez, J.M. $\stackrel{a}{\text { a }}$ Martí, J.M. $\stackrel{a}{ }$, \& MacFadyen, A.I. 2000, ApJL, 531, L119

Akiyama, S., Wheeler, J.C., Meier, D.L., \& Lichtenstadt, I. 2002, ApJ, in press (astro-ph/0208128)

Bailyn, C.D., Jain R.K., Coppi, P., \& Orosz, J.A. 1998, ApJ, 499, 367

Balbus, S.A. \& Hawley, J.F. 1998, Reviews of Modern Physics, 70, 1

Baron, E., Bethe, H.A., Brown, G.E., Cooperstein, J., \& Kahana, S. 1987, Phys. Rev. Lett., 59, 736

Baron, E. \& Cooperstein, J. 1990, ApJ, 353, 597

Baron, E., Cooperstein, J., \& Kahana, S. 1985, Phys. Rev. Lett., 55, 126

Bethe, H.A. 1990, Rev. Mod. Phys., 62, 801

Bethe, H.A. \& Wilson, J.R. 1985, ApJ, 295, 14 (1985)

Bisnovatyi-Kogan, G.S. 1971, Soviet Astronomy AJ, 14, 652

Blandford, R.D. \& Znajek, R.L. 1977, MNRAS, 179, 433

Blondin, J.M., Mezzacappa, A., \& DeMarino, C. 2002, ApJ, in press (astro-ph/0210634)

Bruenn, S.W. 1985, ApJ Supp., 58, 771

Bruenn, S.W. 1989a, ApJ, 340, 955

Bruenn, S.W. 1989b, ApJ, 341, 385

Bruenn, S.W. 1993, in Nuclear Physics in the Universe, ed. M.W. Guidry \& M.R. Strayer (Bristol: IOP) 31

Bruenn, S.W., De Nisco, K.R., \& Mezzacappa, A. 2001, ApJ, 560, 326

Buras, R., Janka, H.-Th., Keil, M.-Th., Raffelt, G., \& Rampp, M. 2002, ApJ, submitted (astro-ph/0205006)

Burrows, A. \& Sawyer, R.F. 1998, Phys. Rev. C., 58, 554

Burrows, A. \& Sawyer, R.F. 1999, Phys. Rev. C., 59, 510

Burrows, A., Hayes, J., \& Fryxell, B.A.. 1995, ApJ, 450, 830

Carter, G.W. \& Prakash, M. 2002, Physics Letters B, 525, 249

Colella, P. \& Woodward, P.R. 1984, J. Computational Physics, 54, 174

Colgate, S.A. \& White, R.H. 1966, ApJ, 143, 626

Cordes, J.M. \& Chernoff, D.F. 1998, ApJ, 505, 315

Daigne, F. \& Mochkovitch, R. 2002, A\&A, 388, 189

Di Matteo, T., Perna, R., \& Narayan, R. 2002, ApJ, 579, 706

Drenkhahn, G. 2002, A\&A, 387, 714 
Drenkhahn, G. \& Spruit, H.C. 2002, A\&A, 391, 1141

Emanuel, K.A. 1991, Ann. Rev. Fluid Mech., 23, 179

Fryer, C.L. 1999, ApJ, 522, 413

Fryer, C.L. \& Heger, A. 2000, ApJ, 541, 1033

Fryer, C.L. \& Warren, M.S. 2002, ApJL, 574, L65

Fryxell, B.A., Müller, E., \& Arnett, W.D. 1989, (Preprint MPA-449, Garching: MaxPlanck-Institut für Astrophysik)

Gotthelf, E.V., Koralesky, B., Rudnick, L., Jones, T.W., Hwang, U., \& Petre, R. 2001, ApJL, 552, L39

Hamuy, M. 2002, ApJ, in press (astro-ph/0209174)

Hannestad, S. \& Raffelt, G. 1998, ApJ, 507, 339

Heger, A., Woosley, S.E., Fryer, C.L., \& Langer, N. 2002, in: From Twilight to Highlight - The Physics of Supernovae, ed. W. Hillebrandt \& B. Leibundgut (Springer Series "ESO Astrophysics Symposia", Berlin: Springer) (astro-ph/0211062)

Einfeldt, B. 1988, SIAM Jour. Numer. Anal., 25, 294

Herant, M. 1995, Physics Rep., 256, 117

Herant, M., Benz, W., \& Colgate, S.A. 1992, ApJ, 395, 642

Herant, M., Benz, W., Hix, W.R., Fryer, C.L., \& Colgate, S.A. 1994, ApJ, 435, 339

Hillebrandt, W., Wolff, R.G., \& Nomoto, K. 1984, A\&A, 133, 175

Höflich, P., Wheeler, J.C., \& Wang, L. 1999, ApJ, 521, 179

Hoffman, R.D., Woosley, S.E., Fuller, G.M., \& Meyer, B.S. 1996, ApJ, 460, 478

Horowitz, C.J. 2002, Phys. Rev. D, 65, 043001-1

Iwamoto, K., et al. 1998, Nature, 395, 672

Janka, H.-Th. 2001, A\&A, 368, 527

Janka, H.-Th. \& Müller, E. 1995, ApJL, 448, L109

Janka, H.-Th. \& Müller, E. 1996, A\&A, 306, 167

Janka, H.-Th., Buras, R., \& Rampp, M. 2002, in Proceedings of the 7th Int. Symposium on Nuclei in the Cosmos, Nuclear Physics A, in press

Keil, W. 1997, PhD Thesis, Technische Universität München

Keil, W., Janka, H.-Th., \& Müller, E. 1996, ApJL, 473, L111

Kifonidis, K. 2002, PhD Thesis, Technische Universität München

LeBlanc, J.M. \& Wilson, J.R. 1970, ApJ, 161, 541

Khokhlov, A.M., Höflich, P., Oran, E.S., Wheeler, J.C., Wang, L., \& Chtchelkanova, A.Yu. 1999, ApJL, 524, L107

Lai, D. 2001, in Physics of Neutron Star Interiors, ed. D. Blaschke, N.K. Glendenning, \& A.D. Sedrakian, (Lecture Notes in Physics, 578, Berlin: Springer) 424

Lai, D., Chernoff, D.F., \& Cordes, J.M. 2001, ApJ, 549, 1111

Lattimer, J.M. \& Swesty, F.D. 1991, Nucl. Phys. A, 535, 331

Leonhard, D.C., Filippenko, A.V., Ardila, D.R., \& Brotherton, M.S. 2001, ApJ, 553, 86

Liebendörfer, M., Messer, O.E.B., Mezzacappa, A., Hix, W.R., Thielemann, F.-K., \& Langanke, K. 2002, in Proc. 11th Workshop on Nuclear Astrophysics, ed. W. Hillebrandt \& E. Müller (Report MPA/P13, Garching: Max-Planck-Institut für Astrophysik) 126 (astro-ph/0203260)

Liebendörfer, M., Mezzacappa, A., Thielemann, F., Messer, O.E.B., Hix, W.R., \& Bruenn, S.W. 2001, Phys. Rev. D., 63, 3004 
Lyne, A.G., \& Lorimer, D.R. 1994, Nature, 369, 127

MacFadyen, A.I. \& Woosley, S.E. 1999, ApJ, 524, 262

MacFadyen, A.I., Woosley, S.E., \& Heger, A. 2001, ApJ, 550, 410

Matzner, C.D. 2002, MNRAS, submitted (astro-ph/0203085)

Mayle, R.W., Tavani, M., \& Wilson, J.R. 1993, ApJ, 418, 398

Meier, D.L., Koide, S., \& Uchida, Y. 2001, Science, 291, 84

Meier, D.L., Epstein, R.I., Arnett, W.D., \& Schramm, D.N. 1976, ApJ, 204, 869

Mezzacappa, A., et al. 2002, in: From Twilight to Highlight - The Physics of Supernovae, ed. W. Hillebrandt \& B. Leibundgut (Springer Series "ESO Astrophysics Symposia", Berlin: Springer)

Mezzacappa, A. \& Bruenn, S.W. 1993, ApJ, 405, 637

Mezzacappa, A. \& Bruenn, S.W. 1993, ApJ, 410, 740

Mezzacappa, A., Liebendörfer, M., Messer, O.E.B., Hix, W.R., Thielemann, F.-K., \& Burenn, S.W. 2001, Phys. Rev. Lett., 86, 1935

Mezzacappa, A., Calder, A.C., Bruenn, S.W., Blondin, J.M., Guidry, M.W., Strayer, M.R., \& Umar, A.S. 1998, ApJ, 495, 911

Mönchmeyer, R. 1993, PhD Thesis, Technische Universität München

Müller, E. \& Hillebrandt, W. 1979, A\&A, 80, 147

Myra, E.S. \& Bludman, S.A. 1989, ApJ, 340, 384

Myra, E.S., Bludman, S.A., Hoffman, Y., Lichenstadt, I., Sack, N., \& van Riper, K.A. 1987, ApJ, 318, 744

Nadyozhin, D.K. 2002, A\&A, submitted (Preprint MPA 1458, Garching: Max-PlanckInstitut für Astrophysik)

Nomoto, K., Shigeyama, T., Kumagai, S., Yamaoka, H., Suzuki, T. 1994, in Supernovae, Les Houches Session LIV, ed. S.A. Bludman, R. Mochkovitch, \& J. ZinnJustin (Amsterdam: Elsevier/North-Holland) 489

Nomoto, K., Maeda, K., Umeda, H., Ohkubo, T., Deng, J., \& Mazzali, P. 2002, in A Massive Star Odyssey, from Main Sequence to Supernova, Proc. IAU Symposium 212, ed. K.A. van der Hucht, A. Herrero, \& C. Esteban (San Francisco: ASP) in press (astro-ph/0209064)

Ostriker, J.P. \& Gunn, J.E. 1971, ApJL, 164, L95

Shapiro, S.L. \& Teukolsky, S.A. 1983, Black Holes, White Dwarfs, and Neutron Stars (New York: Wiley)

Plewa, T. \& Müller, E. 1999, A\&A, 342, 179

Plewa, T. \& Müller, E. 2001, Computer Physics Communications, 138, 101

Popham, R., Woosley, S.E., \& Fryer, C.L. 1999, ApJ, 518, 356

Quirk, J.J. 1994, Int. J. Num. Meth. Fluids, 18, 555

Rampp, M. \& Janka, H.-Th. 2000, ApJL, 539, L33

Rampp, M. \& Janka, H.-Th. 2002, A\&A, 396, 361

Shimizu, T., Yamada, S., \& Sato, K. 1993, Publ. Astron. Soc. Japan, 45, L53

Shimizu, T., Yamada, S., \& Sato, K. 1994, ApJL, 432, L119

Shimizu, T.M., Ebisuzaki, T., Sato, K., \& Yamada, S. 2001, ApJ, 552, 756

Stairs, I.H., Thorsett, S.E., Taylor, J.H., \& Wolszczan, A. 2002, ApJ, in press (astro-ph/0208357)

Sumiyoshi, K., Terasawa, M., Mathews, G.J., Kajino, T., Yamada, S., \& Suzuki, H. 2001, ApJ, 562, 880

Swesty, F.D., Lattimer, J.M., \& Myra, E.S. 1994, ApJ, 425, 195 
Thompson, C. \& Duncan, R.C. 1993, ApJ, 408, 194

Thompson, C. \& Murray, N. 2001, ApJ, 560, 339

Thompson, T.A., Burrows, A., \& Pinto, P.A. 2002, ApJ, submitted (astro-ph/0211194)

Thorsett, S.E. \& Chakrabarty, D. 1999, ApJ, 512, 288

Thorstensen, J.R., Fesen, R.A., \& van den Bergh, S. 2001, ApJ, 122, 297

Totani, T., Sato, K., Dalhed, H.E., \& Wilson, J.R. 1998, ApJ, 496, 216

Wang, L., Howell, D.A., Höflich, P., \& Wheller, J.C. 2001, ApJ, 550, 1030

Wang, L., Wheeler, J.C., Höflich, P., Khokhlov, A., et al. 2002, ApJ, in press (astro-ph/0205337)

Wheeler, J.C. 2002, AAPT/AJP Resource Letter, American J. of Physics, in press (astro-ph/0209514)

Wheeler, J.C., Meier, D.L., \& Wilson, J.R. 2002, ApJ, 568, 807

Wheeler, J.C., Yi, I., Höflich, P., \& Wang, L. 2000, ApJ, 537, 810

Wilson, J.R. 1985, in Numerical Astrophysics, ed. J.M. Centrella, J.M. LeBlanc, R.L. Bowers, \& J.A. Wheeler (Boston: Jones and Bartlett) 422

Wilson, J.R. \& Mayle, R. 1988, Phys. Rep., 163, 63

Wilson, J.R. \& Mayle, R. 1993, Phys. Rep., 227, 97

Woosley, S.E. 1993, ApJ, 405, 273

Woosley, S.E., Heger, A., \& Weaver, T.A. 2002, Reviews of Modern Physics, in press Woosley, S.E., Zhang, W., \& Heger, A. 2002, in: From Twilight to Highlight - The Physics of Supernovae, ed. W. Hillebrandt \& B. Leibundgut (Springer Series "ESO Astrophysics Symposia", Berlin: Springer) (astro-ph/0211063)

Zhang, W., Woosley, S.E., \& MacFadyen, A.I. 2002, ApJ, submitted (astro-ph/0207436) 